\title{
Encefalitis y abscesificación cerebral como complicación de una sinusitis frontal aguda en un niño
}

\author{
Encephalitis and brain abscesification as a complication of acute frontal \\ sinusitis in a child
}

\author{
Clara Espina G. ${ }^{1}$, Felip Ferrer B. ${ }^{1}$, Delfina Dualde B. ${ }^{2}$, María Aragonés R. $^{1}$, Jaime Marco A. ${ }^{1}$
}

\section{Resumen}

Las sinusitis son procesos infecciosos-inflamatorios de las cavidades neumáticas paranasales. Entre las complicaciones de las sinusitis agudas se encuentra la afectación intracraneal, con meningoencefalitis e incluso abscesos extra o subdurales. En este trabajo se describe un caso de meningoencefalitis y empiemas subdurales derivados de una sinusitis frontal aguda en un niño de 12 años, que acudió a urgencias presentando alteración del nivel de conciencia y hemiparesia derecha. Se realizan estudios de tomografía computada y resonancia magnética cerebral, evidenciándose ocupación de seno frontal izquierdo, acompañada de extensa afectación hipercaptante en cubiertas en hemisferio cerebral izquierdo, con formación de empiemas subdurales a nivel frontal izquierdo, parafalciano y en reborde tentorial. Ingresa para tratamiento antibiótico intravenoso y dado el nivel de afectación y la escasa respuesta a tratamiento médico, se decide realizar cirugía endoscópica DRAF IIa con drenaje del absceso frontal, etmoidectomía y meatotomía media izquierda, con sonda de drenaje a fosa nasal. Las complicaciones a nivel de sistema nervioso central son graves y han de tenerse siempre presentes, requiriendo en ocasiones drenaje quirúrgico con abordaje por vía endonasal o abierta.

Palabras clave: Sinusitis frontal, encefalitis, empiema, DRAF IIa.

\begin{abstract}
Sinusitis are infectious-inflammatory processes of the pneumatic paranasal cavities. Among the complications of acute sinusitis, we can find intracranial involvement, with meningoencephalitis and even extra or subdural abscesses. In this article we describe a case of meningoencephalitis and subdural empyemas associated with acute frontal sinusitis in a 12-year-old child who presented to the emergency room referring altered level of consciousness and right hemiparesis. Computed tomography scan and brain magnetic resonance imaging studies were performed, evidencing left frontal sinus occupation, accompanied by extensive hypercaptant defects in the left cerebral hemisphere, with formation of subdural empyemas at the left frontal level, parafalcian and in the tentorial area. He was hospitalized for intravenous antibiotic treatment, and given the lack of response to medical treatment, a DRAF IIa sinus surgery and drainage of the frontal abscess, ethmoidectomy and left middle meatotomy with drainage tube to the nasal cavity were performed. Complications at the central nervous system are serious and must always be kept in mind, sometimes requiring surgical drainage with an endonasal or open approach.
\end{abstract}

Keywords: frontal sinusitis, encephalitis, empiema, DRAF IIa.
${ }^{1}$ Servicio de Otorrinolaringología y Cirugía de Cabeza y Cuello. Hospital Clínico Universitario Valencia, Universidad de Valencia. Valencia, España.

${ }^{2}$ Servicio Radiodiagnóstico. Hospital Clínico Universitario Valencia, Universidad de Valencia. Valencia, España.

Los autores declaran no tener conflictos de interés.

Recibido el 18 de mayo de 2020. Aceptado el 1 de diciembre de 2020 .

\section{Correspondencia:}

Clara Espina G.

Hospital Clínico Universitario Avenida Blasco Ibáñez 17, 46010

Valencia, España. Email: claraespina8@gmail. com.

\section{Caso Clínico}

Paciente varón de 12 años sin antecedentes personales ni familiares de interés, correctamente vacunado para su edad. Acudió a urgen- cias de nuestro hospital presentando alteración del nivel de conciencia, hemiparesia derecha y fiebre de $40^{\circ} \mathrm{C}$. Ante la clínica de menos de 4 horas y media de hemiparesia, se activó el código ictus y se realizó una tomografía com- 
putada (TC) que evidenció una ocupación de senos frontal y maxilar izquierdo, y angio-TC sin alteraciones (Figura 1A).

La resonancia magnética (RM) mostró ocupación de seno frontal izquierdo, afectación hipercaptante en cubiertas en hemisferio cerebral izquierdo, empiemas subdurales a nivel frontal izquierdo, parafalciano y en reborde tentorial. Se realizó punción lumbar, compatible con meningitis bacteriana aguda, por lo que se inició tratamiento intravenoso antibiótico inicialmente con cefotaxima y vancomicina. Dado que no presentó mejoría, a los 2 días se cambió por meropenem y linezolid.

El nuevo tratamiento médico se mantuvo 48 horas, sin embargo, ante la ausencia de mejoría clínica ni en las pruebas complementarias, el paciente fue intervenido para realización de un drenaje del seno frontal mediante cirugía endoscópica nasosinusal (CENS) izquierda con abordaje mediante neuronavegador del seno frontal izquierdo (DRAF IIA), realizando et- moidectomía y meatotomía media ipsilateral, colocando sonda de drenaje suturada al septum. Los resultados de la muestra quirúrgica de seno frontal mostraron Staphylococcus aureus meticilina sensible y Candida albicans, por lo que se ajustó el tratamiento a cefotaxima, anfotericina B y metronidazol.

Se realizó RM con venografía tras el drenaje quirúrgico, que confirmó la presencia de una trombosis venosa cerebral, y se inició tratamiento con heparina de bajo peso molecular. Diez días tras la cirugía, el paciente refirió cefalea intensa y cefalea occipital, con movilidad cervical no dolorosa, manteniéndose en todo momento afebril. La RM evidenció aumento de tamaño de los empiemas subdurales de localización infra y supratentorial y convexidad izquierda, con desviación de línea media y colapso parcial del ventrículo lateral izquierdo (Figura 1B, C y D) por lo que fue intervenido por neurocirugía. Se realizó una craniectomía fronto-temporo-parietal izquierda, con drena-

Figura 1. Tomografía computada y resonancia magnética cerebral preoperatorias. A) Corte axial TC de senos paranasales que muestra ocupación bilateral de senos frontales y etmoidales. B) Secuencia axial de RM difusión con áreas de captación en los empiemas. C y D) Cortes axiales de RM donde se evidencian dos focos de empiema.
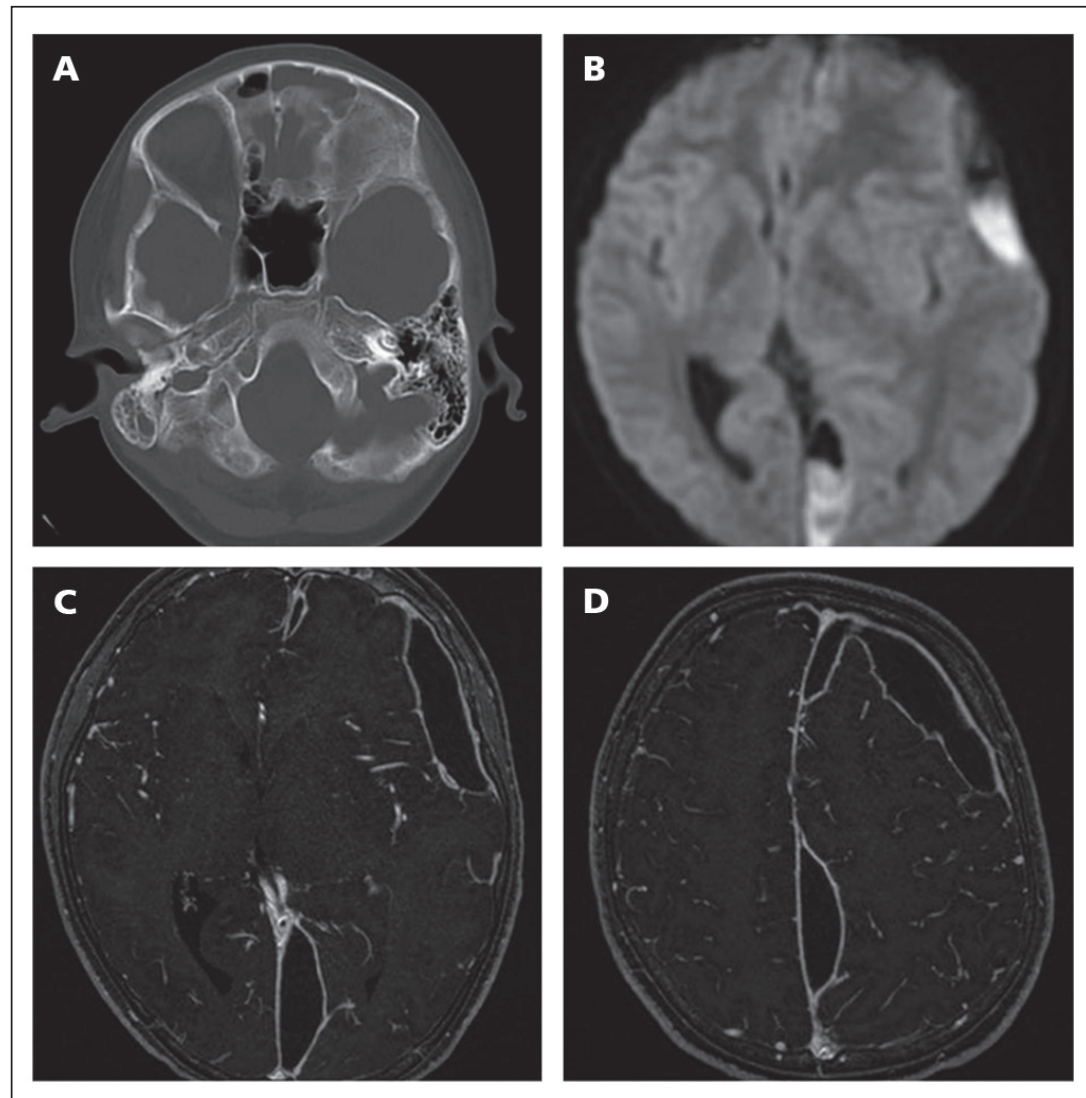
je de material purulento en espacio subdural a ese nivel, e interhemisférico, dejando drenajes Jackson Pratts, tras lo cual se produjo una mejoría clínica progresiva del paciente.

Se realizó una RM de control el décimo día posoperatorio, que mostró aumento de tamaño de los empiemas subdurales ya conocidos, infratentorial derecho, supratentorial temporal izquierdo y parafalciano también izquierdo. Con estos resultados, se decidió reintervenir por parte de neurocirugía, para nueva evacuación. Tras la segunda evacuación, se produjo una mejoría total de los parámetros clínico-radiológicos. El paciente fue dado de alta asintomático. A los 2 meses de seguimiento se retiró drenaje (Figura 2). Un año tras la intervención, el paciente se mantiene asintomático y se aprecia bien canalizado el acceso al seno frontal, sin presentar ninguna secuela.

\section{Discusión}

Según la guía EPOS 2020 (European positioning paper on rhinosinusitis and nasal polyps 2020), las complicaciones de la rinosinusitis bacteriana aguda en los niños se clasifican en orbitarias $(60 \%-80 \%)$, intracraneales $(15 \%$ $20 \%)$ y óseas (5\%). A su vez las orbitarias se dividen según la clasificación de Chandler en 5 tipos según su localización y nivel de afectación ${ }^{1}$.

En cuanto a las complicaciones intracraneales derivadas de una sinusitis con complicaciones poco frecuentes, pero potencialmente graves $^{2}$, las complicaciones craneales más frecuentes son por orden de frecuencia: absceso epidural, empiema subdural, meningitis, encefalitis y absceso cerebral, como en el caso clínico anteriormente descrito ${ }^{3}$. El diagnóstico y manejo de estos pacientes es complejo, y requiere la colaboración de distintos especialistas: otorrinolaringólogos, pediatras, neurocirujanos, neurólogos e internistas entre otros.

Las colecciones subdurales y epidurales secundarias a sinusitis se producen más frecuentemente en niños mayores, adolescentes varones ${ }^{4}$, como en el caso de nuestro paciente. Los abscesos epidurales se forman entre el cráneo y la duramadre y se expanden lentamente debido a la estrecha adherencia de la duramadre al hueso. Su presentación clínica

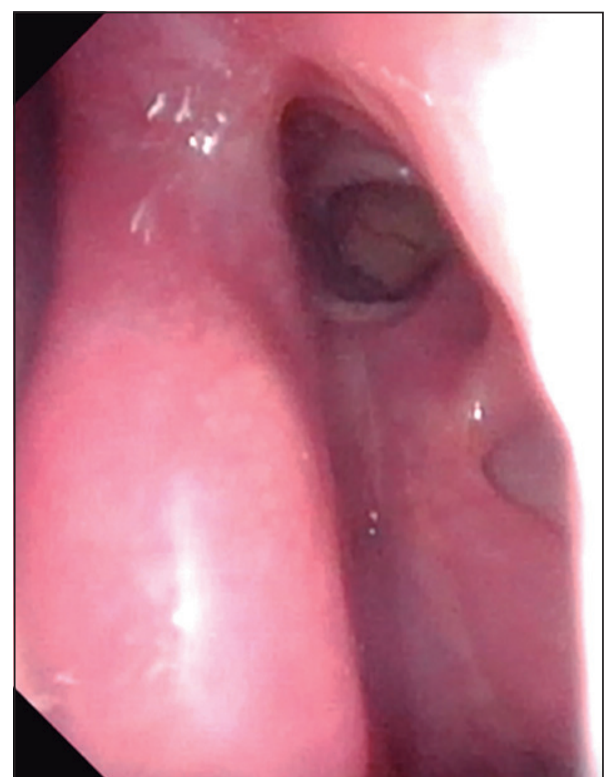

Figura 2. Neoostium del seno frontal. Visión endoscópica en visita de control del neoostium del seno frontal tras el drenaje DRAF IIA.

es típicamente insidiosa (hasta varias semanas), con un período prolongado de síntomas inespecíficos. Por el contrario, el empiema subdural se propaga rápida y libremente dentro de un espacio preformado, lo que generalmente resulta en una presentación aguda más fulminante que con el absceso epidural, con un desarrollo más temprano de déficits neurológicos. Es igualmente probable que los abscesos intracerebrales se presenten con o sin signos focales. En complicaciones intracraneales, es común la escasez de síntomas y signos focales (afectación intracraneal "silenciosa"), especialmente en los niños.

La resonancia magnética se considera superior al TC con contraste en la detección de colecciones intracraneales, y su capacidad para detectar la inflamación meníngea es útil para confirmar la meningitis ${ }^{5}$. La sensibilidad adicional de la RM permite un diagnóstico más precoz de abscesos más pequeños.

El tratamiento médico requiere tratamiento intravenoso "agresivos" con antibióticos que atraviesen la barrera hematoencefálica y con cobertura antibiótica frente a cocos gram positivos. En este caso debido a la coinfección por Staphylococcus aureus meticilino sensible 
y Candida albicans, se trató con cefotaxima, anfotericina B y metronidazol.

Citando nuevamente la guía EPOS 2020, el tratamiento de las complicaciones de tipo intracraneal debe realizarse mediante antibioterapia intravenosa, como se ha explicado previamente, acompañada de drenaje quirúrgico por parte de otorrinolaringólogos y neurocirujanos, tratando mediante cirugía endoscópica los senos afectados lo antes posible, como se produjo en el caso descrito, y craneotomía si se considera necesario ${ }^{6-8}$.

En el caso que presentamos se decidió realizar un manejo quirúrgico para tratar la sinusitis frontal por parte de otorrinolaringología. Se efectuó un drenaje suficientemente amplio del seno frontal izquierdo y se utilizó neuronavegador para tener más seguridad en el acceso. Ante la persistencia del cuadro y empeoramiento de las colecciones intracerebrales, a pesar de que permanecía permeable y sin drenaje purulento el abordaje endoscópico del seno frontal se reintervino por parte de neurocirugía en dos ocasiones. La coinfección de una rinosinusitis bacteriana con infección fúngica, en este caso por Candida albicans, no es frecuente, aunque está descrita asociada a largas pautas de antibioterapia. En las rinosinusitis crónicas, el Staphylococcus aureus es el patógeno más frecuente ${ }^{9,10}$. La colonización por hongos se ha estudiado como factor patogénico asociado a la inflamación en rinosinusitis crónicas ${ }^{11}$.

En conclusión, la afectación intracraneal forma parte de las complicaciones que, aunque son poco frecuentes, pueden poner en compromiso la vida del paciente. Es importante una alta sospecha clínica y un correcto manejo multidisciplinario.

\section{Bibliografía}

1. Fokkens WJ, Lund VJ, Hopkins C, Hellings PW, Kern R, Reitsma S, Toppila-Salmi S, Bernal-Sprekelsen
M, Mullol J, Alobid I, et al. European Position Paper on Rhinosinusitis and Nasal Polyps 2020. Rhinology. 2020;58(Suppl S29):1-464. doi: 10.4193/Rhin20.600.

2. Germiller JA, Monin DL, Sparano AM, Tom LWC. Intracranial Complications of Sinusitis in Children and Adolescents and Their Outcomes. Arch Otolaryngol Head Neck Surg. 2006;132(9):969-976. doi:10.1001/archotol.132.9.969.

3. Hibi, A. and Amakusa, Y. Intracranial subdural abscess with polymicrobial infections due to frontal sinusitis in an adolescent: life-threatening complication of a common disease. Clin Case Rep. 2018, 6: 516-521. doi:10.1002/ccr3.1355.

4. M.P. Martínez Beneito, L. De La Fuente Arjona, F.J. Garć́a Callejo, I. Mallea Cañizares, L. Blay Galaud, J. Marco Algarra, Empiema subdural de causa sinusal por gemela morbillorum, una extraña etiología. Acta Otorrinolaringol Esp. 2002;53(6):427-430. doi: 10.1016/S0001-6519(02)78332-5.

5. Martines F, Salvago P, Ferrara S, Mucia M, Gambino A, Sireci F. Parietal subdural empyema as complication of acute odontogenic sinusitis: a case report. J Med Case Rep. 2014;8:282. doi:10.1186/17521947-8-282.

6. Turner JH, Vaezeafshar R, Hwang PH. Indications and outcomes for Draf IIB frontal sinus surgery. Am J Rhinol Allergy. 2016;30(1):70-73. doi:10.2500/ ajra.2016.30.4268.

7. Draf W. Endonasal Frontal Sinus Drainage Type IIII According to Draf. In: Kountakis S, Senior B, Draf W. (eds) The Frontal Sinus. 2016 Springer, Berlin, Heidelberg Springer, Berlin, Heidelberg.

8. Martín-Hernández G, Collazo-Lorduy T, VelascoGarcía MJ, Gavilanes-Plasencia J, SánchezJara JL. Revisión del tratamiento quirúrgico de la rinosinusitis. Acta Otorrinolaringol Esp. 2011;62(1):56-64. doi: 10.1016/j.otorri.2010.01.007.

9. Cabrera-Maqueda JM, Fuentes Rumí L, Valero López G, et al. Difusión de los antibióticos en el sistema nervioso central. Rev Esp Quimioter. 2018;31(1):1-12.

10. Stephenson MF, Mfuna L, Dowd S, Wolcott R, Barbeau J, Poisson M, et al. Molecular Characterization of the Polymicrobial Flora in Chronic Rhinosinusitis. J Otolaryngol Head Neck Surg. 2010;39:182-7.

11. Slavin RG. Sinusitis: Viral, bacterial, or fungal and what is the role of staph? Allergy Asthma Proc. 2006;27(6):447-50. 